\title{
Características de los perros y gatos bajo control reproductivo quirúrgico registrados en la Municipalidad de Los Olivos, Lima, Perú. Periodo 2015-2016
}

\author{
Characteristics of dogs and cats under surgical reproductive control registered in \\ the Municipality of Los Olivos, Lima, Peru. Period 2015-2016 \\ Priscila Rojas V. ${ }^{1}$, Daphne León C. ${ }^{1}$, Néstor Falcón P. ${ }^{1,2}$
}

\section{Resumen}

\begin{abstract}
El objetivo del estudio fue describir las características demográficas de la población de animales de compañía que fueron sometidos a control reproductivo quirúrgico en la Municipalidad de Los Olivos (Lima, Perú) en el periodo 2015-2016. Se revisaron los registros de la Clínica Veterinaria Municipal de Los Olivos recolectando información de las variables edad, sexo, especie y dirección del propietario. La información fue analizada mediante estadística descriptiva. Se obtuvo 140 fichas de perros y 155 de gatos esterilizados. La media de edad de los perros fue de $2.1 \pm 1.5$ (rango: 6 meses a 7 años) y de los gatos fue de $1.2 \pm 0.8$ (rango: 6 meses a 4 años). El mayor porcentaje de esterilizaciones correspondió a las hembras en ambas especies (90.7\% en perros y $89.0 \%$ en gatos) y de raza mestiza (93.6\% en perros y $99.4 \%$ en gatos). El área de influencia de la Clínica Veterinaria Municipal de Los Olivos tuvo un radio de hasta $8.26 \mathrm{~km}$. Se espera que la información sea de utilidad para determinar la población objetivo de futuras campañas de esterilización, así como planificar necesidades logísticas que se han de requerir.
\end{abstract}

Palabras clave: esterilización; Los Olivos; control poblacional; zoonosis

\section{Abstract}

The aim of this study was to describe the demographic characteristics of the population of companion animals that underwent surgical reproductive control in the Municipality of Los Olivos (Lima, Peru) in the 2015-2016 period. The records of the Los Olivos Municipal Veterinary Clinic were reviewed by collecting information on the age,

\footnotetext{
${ }^{1}$ Grupo de Salud Pública Veterinaria (SAPUVET-PERÚ), Facultad de Medicina Veterinaria y Zootecnia, Universidad Peruana Cayetano Heredia, Lima, Perú

${ }^{2}$ E-mail: nestorfalcon@upch.pe
} 
sex, species and address of the owner. The information was analyzed by descriptive statistics. Records of $140 \mathrm{dog}$ and 155 cats were obtained. The average age of the dogs was $2.1 \pm 1.5$ (range: 8 months to 7 years) and of the cats was $1.2 \pm 0.8$ (range: 6 months to 4 years). The highest percentage of sterilizations corresponded to females in both species $(90.7 \%$ in dogs and $89.0 \%$ in cats) and of mestizo phenotype (93.6\% in dogs and $99.4 \%$ in cats). The area of influence of the Municipal Veterinary Clinic of Los Olivos had a radius of up to $8.26 \mathrm{~km}$. It is expected that the information will be useful to determine the target population of future neutering campaigns, as well as to plan logistics needs that will be required.

Key words: neutered; Los Olivos; population control; zoonoses

\section{INTRODUCCIÓN}

La población de animales de compañía, llámese perros y gatos, ha ido aumentando con el paso de los años (Miró et al., 2003), aunque se desconoce el número exacto debido a que no hay datos actualizados. En Perú, Málaga (2010) menciona resultados de los primeros estudios de Lima Metropolitana realizados en 1970, 1974 y 1985, donde obtuvo la relación persona: perro de 10:1, 11:1 y 11:1, respectivamente. Estudios más actuales reportan una relación persona: perro de 7.8:1 para la ciudad de Lima (Llalla, 2012), 7:1 para San Martín de Porres (Arauco et al., 2014) y de 5.7:1 para el distrito de Comas (Soriano et al., 2017).

La población de perros vagabundos en el distrito de Los Olivos, Lima, que deambulaban en las calles en horario diurno fue estimada en $1411 \pm 643$ perros y en horario nocturno de $922 \pm 497$ (Ochoa et al., 2014). En Villa El Salvador, Lima, asimismo, se estimó que existían $24.6 \pm 4.9$ y $27.1 \pm 7.7$ perros en el interior y exterior de 21 mercados formales (Chávez et al., 2014).

Respecto a la población de gatos con dueño en Lima Metropolitana, se menciona una relación de persona: gato de 18.4:1 (Lla1la, 2012), mientras que en el distrito de San Borja, Lima, se halló una relación de 5:1 (Arellano et al., 2018) y en Ventanilla, Callao, se halló la relación de 4:1 en viviendas ubicadas en asentamientos humanos (Rendón et al., 2018).

El crecimiento de la población de animales de compañía en estado de abandono estaría relacionado con problemas económicos y la falta de sensibilización sobre tenencia responsable hacia los propietarios, lo que trae como consecuencia la presencia de animales en las calles y una reproducción sin control (FAO, 2014). El crecimiento de la población de animales de compañía tiene un efecto directo en la salud humana, ya que pueden transmitir enfermedades zoonóticas, contaminar el ambiente y causar accidentes por mordedura de perros (León et al., 2013).

Existen diversas estrategias para el control de la población de animales de compañía, siendo las esterilizaciones de hembras y machos la opción más utilizada (FAO, 2014). Estas pueden ser quirúrgicas (Root-Kustritz, 2012) o no quirúrgicas (FAO, 2014). Sin embargo existen otras alternativas como la educación, sensibilización (FAO, 2014) y la separación física (WHO y WSPA, 1990; ICAM, 2007). Por último, como opción más drástica se contempla a la eutanasia (Consejo Canadiense de Protección de los Animales, 1998; Tasker, 2013).

El control de la población de animales de compañía en muchos casos es asumido como una responsabilidad de las municipalidades. En este contexto, la municipalidad limeña de Los Olivos viene desarrollando 
campañas de esterilización quirúrgicas desde 2011. La información de las campañas no ha sido sistematizada y se requiere evaluar la cantidad de animales que han sido esterilizados y sus características, a fin de prever las necesidades logísticas y su proyección para el futuro. Por ello, el objetivo del estudio fue describir las características demográficas de la población de animales de compañía que han sido sometidos a control reproductivo quirúrgico en esta comuna en el periodo 20152016.

\section{Materiales y MéTodos}

El estudio descriptivo retrospectivo recolectó información de las fichas de esterilizaciones de la Clínica Veterinaria Municipal del distrito de Los Olivos para el periodo 20152016. El procesamiento y análisis de datos se realizó en la Facultad de Medicina Veterinaria y Zootecnia de la Universidad Peruana Cayetano Heredia (FAVEZ-UPCH), Lima.

El programa de esterilización de la Municipalidad de Los Olivos aceptaba a todos los perros y gatos en estado fisiológico adecuado para ser sometidos a cirugía. Además, el animal debía tener un propietario que se haga responsable. La cirugía se ofrecía a una tarifa social con el fin de cumplir con lo estipulado en la Ley №27596.

El estudio incluyó todas las fichas de esterilización de perros y gatos que tenían información completa y letra legible. Se excluyó del estudio aquellas fichas que no consideraban la información demográfica de los pacientes y aquellas que correspondían a animales cuyos propietarios no residían en el distrito de Los Olivos.

La información recolectada correspondió a las variables: especie, edad, raza, tamaño y sexo de los animales de compañía y, además, la dirección del propietario. Se generó una base de datos en Microsoft Excel y el análisis se realizó con el programa Stata
13.0. Las variables cuantitativas se resumieron a través de medidas de tendencia central (media) y medidas de dispersión (rango y desviación estándar). Las variables cualitativas se resumieron a través de tablas de frecuencias.

Para determinar el área de influencia geográfica del servicio de esterilizaciones de la clínica se utilizó la información de las direcciones de los propietarios de los animales esterilizados. Con ellas se obtuvo puntos GPS mediante la herramienta Google Earth y se elaboró un mapa que indicaba el punto de ubicación de la clínica y la distribución de los puntos correspondiente al domicilio de los animales esterilizados. En esta etapa, se excluyeron las fichas que no contenían la información completa de la dirección del propietario del animal de compañía para poder generar los puntos GPS.

El proyecto fue aprobado por el Comité Institucional de Ética de la UPCH y se mantuvo la confidencialidad de la información recolectada.

\section{Resultados}

La Clínica Veterinaria Municipal de Los Olivos proporcionó 371 fichas de esterilizaciones realizadas en el periodo del estudio, quedando finalmente 295 fichas que cumplieron los criterios de inclusión. De estas, 140 fichas fueron de perros y 155 de gatos. La edad de los perros esterilizados fue de $2.1 \pm$ 1.5 y un rango de 6 meses y 7 años. Los gatos esterilizados tuvieron una edad de 1.2 \pm 0.8 y un rango de edad entre 6 meses y 4 años. Hubo 51 fichas de perros y 62 de gatos que no contenían información sobre la edad. Así mismo, una ficha de gatos no contenía información sobre el sexo del animal esterilizado. La descripción de la población de perros y gatos esterilizados según variables incluidas en el estudio se detalla en los Cuadros 1 y 2 . 
Cuadro 1. Distribución proporcional de los perros esterilizados en la Clínica Veterinaria Municipal de Los Olivos en el periodo 2015 - 2016, según sexo y raza

\begin{tabular}{clcc}
\hline Variable & Estratos & Número & Porcentaje \\
\hline Año & 2015 & 76 & 54.3 \\
$(\mathrm{n}=140)$ & 2016 & 64 & 45.7 \\
Sexo & Hembra & 127 & 90.7 \\
$(\mathrm{n}=140)$ & Macho & 13 & 9.3 \\
Estrato etario & 6 meses a $<1$ año & 19 & 21.3 \\
$(\mathrm{n}=89)$ & $1-3$ años & 42 & 47.2 \\
& $>3$ años & 28 & 31.5 \\
Raza & Mestizo & 131 & 93.6 \\
$(\mathrm{n}=140)$ & Definida* & 9 & 6.4 \\
\hline
\end{tabular}

*Razas definidas: Perro Peruano sin Pelo (2), Shi-Tzu (1), Pastor Alemán (1), Pekinés (1), Schnauzer (1), Cocker (1), Dogo Argentino (1), Poodle (1)

Cuadro 2. Distribución proporcional de los gatos esterilizados en la Clínica Veterinaria Municipal de Los Olivos en el periodo 2015 - 2016, según sexo y raza

\begin{tabular}{clcc}
\hline Variable & Estratos & Número & Porcentaje \\
\hline Año & 2015 & 90 & 58.1 \\
$(\mathrm{n}=155)$ & 2016 & 65 & 41.9 \\
Sexo & Hembra & 137 & 89.0 \\
$(\mathrm{n}=154)$ & Macho & 17 & 11.0 \\
Estrato etario & 6 meses a $<1$ año & 38 & 40.9 \\
$(\mathrm{n}=93)$ & $1-3$ años & 48 & 51.6 \\
& $>3$ años & 7 & 7.5 \\
Raza & Mestizo & 154 & 99.4 \\
$(\mathrm{n}=155)$ & Siamés & 1 & 0.6 \\
\hline
\end{tabular}

Para la elaboración del mapa se utilizaron 155 datos, que correspondieron a los registros que indicaban una dirección de domicilio exacta que permita generar los puntos GPS. La distribución general de los domicilios alrededor de la clínica tuvo un radio desde $1.0 \mathrm{~km}$ hasta $8.3 \mathrm{~km}$ (Figura 1). Además, el $22 \%$ (65) de las viviendas de animales estu- vieron agrupadas en un radio de $0.94 \mathrm{~km}$ (Figura 2). Se logró ubicar tres puntos (domicilios) que contenían varios registros de esterilizaciones, 2 con 14 y 1 con 8 . Estos domicilios fueron visitados para entrevistar a los propietarios quienes mencionaron que solo ayudaban a animales de la calle, mas no contaban con un lugar específico donde mantenerlos. 


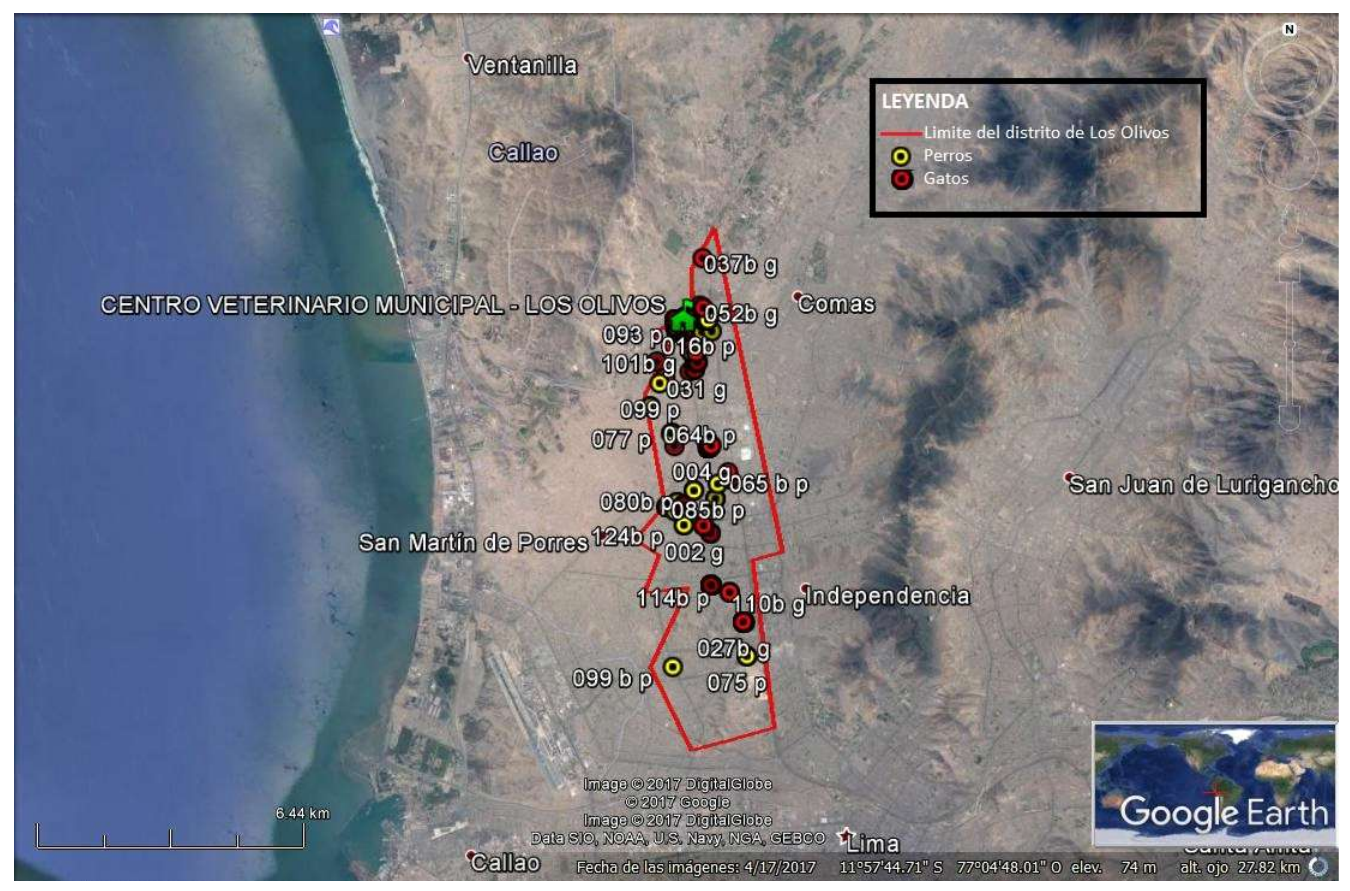

Figura 1. Distribución general de domicilios de animales de compañía esterilizadas en la Clínica Veterinaria Municipal de Los Olivos (periodo 2015 y 2016)

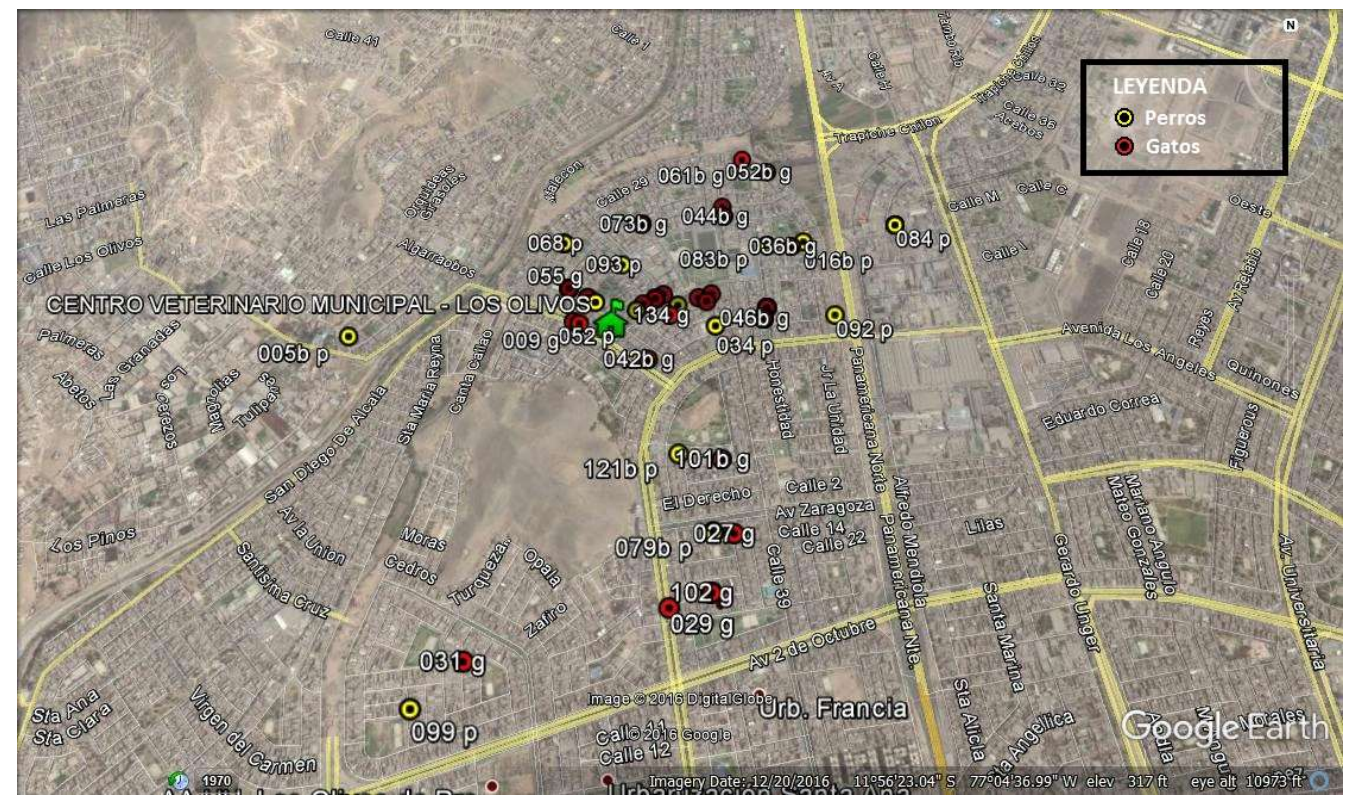

Figura 2. Distribución de domicilios de animales de compañía esterilizados en un radio de 0.94 km de la Clínica Veterinaria Municipal de Los Olivos (periodo 2015 y 2016) 


\section{Discusión}

El estudio muestra que el servicio de esterilizaciones fue requerido en mayor número para gatas que para perras, probablemente debido a las diferencias en la fisiología reproductiva de ambas especies, toda vez que la gata es una una especie poliéstrica estacional (Monstserrat, 2015). Además, si al parir se encuentra en la estación reproductiva entrará en celo a las cuatro semanas del parto, y en el caso de aborto o de ser separadas de las crías al nacer, el celo se presenta nuevamente a los 4-6 días, lo que abre la posibilidad a quedar preñada nuevamente (Giménez et al., 2006). Por el contrario, la perra es una especie monoestral no estacional (Monstserrat, 2015), y por lo tanto, la frecuencia de celo y probabilidad de quedar preñada es bastante menor.

Cuando una gata va a entrar en celo, muestra un comportamiento afectuoso, presenta vocalizaciones y es menos hostil con el macho, y durante el celo aumentan las vocalizaciones (Giménez et al., 2006), lo cual genera más complicaciones y el proceso se puede volver más molesto para el dueño. Sin embargo, los resultados del estudio difieren de lo mencionado por Lui et al. (2011), quienes indican que en una campaña de esterilización realizada en Jaboticabal, Brasil, desde mediados de 2007 hasta fines de 2009 fueron atendidos 1714 animales de los cuales el $54 \%(n=926)$ fueron perros y $46 \%$ $(n=788)$ fueron gatos.

Las esterilizaciones fueron dirigidas mayoritariamente a las hembras en ambas especies. La tendencia de esterilizar hembras podría deberse a razones culturales. Existen propietarios que no toman como opción esterilizar a los machos debido a que consideran que estos pueden «tener vergüenza» (Salamanca et al., 2011), además que tienden a subir de peso (Miller, 2012). Por otro lado, cuando las hembras quedan preñadas serán ellas las que lleven a casa a las crías, hasta que estas puedan sostenerse por sí mismas, lo que implica gastos, preocupación e incomodidad en los propietarios. Por lo general, la responsabilidad del cuidado de los cachorros recae en los propietarios de las hembras, quienes probablemente pueden abandonar a las crías al crecer. En el estudio de Lui et al. (2011) se observó una tendencia similar, pues en perros el $81.1 \%(\mathrm{n}=751)$ eran hembras y en gatos el 57\% $(n=449)$ eran hembras.

Existe controversia sobre la edad a la que debe ser esterilizado un animal de compañía. En la década de los 80 's se optaba por esterilizar a los 5-9 meses de edad para evitar complicaciones con la anestesia; sin embargo, con la mejora de los protocolos de anestesia, la edad para esterilizar disminuyó a las 6-14 semanas (Sontas et al., 2012). Se considera que la esterilización antes del desarrollo hormonal en la hembra disminuye el riesgo de piometras o tumores mamarios, mientras que en los machos disminuye la posibilidad de desarrollar neoplasias prostáticas y controla el comportamiento (Howe, 2006).

Spain et al. (2004b) afirman que en perros no hay consecuencias negativas si se opera al macho antes de los cinco meses. Por otro lado, una esterilización temprana en gatos les cambia el comportamiento, los hace más tímidos comparados a un animal esterilizado más allá de los seis meses (Spain et al., 2004a). La castración en los machos les reduce la agresividad, el comportamiento sexual y el olor de la orina (Spain et al., 2004a,b). Por otro lado, también se considera que no hay beneficios en la esterilización prepuberal y que la esterilización quirúrgica no es necesaria, excepto en aquellos animales que presenten patologías en los órganos reproductores (Reichler, 2009; Root-Kustritz, 2014). La esterilización prepuberal puede aumentar la probabilidad de causar incontinencia urinaria y dermatitis perivulvar en hembras, por lo que se recomienda realizarla después de los cinco meses de edad, pero antes del primer celo (Spain et al., 2004a,b; Root, 2010). 
En la Clínica Veterinaria Municipal de Los Olivos se observó una mayor cantidad de animales esterilizados en el rango de 1 a 3 años. En esta situación, se podría optar por realizar la esterilización antes de los seis meses. Esta opción podría hacer más eficiente el control poblacional, ya que las hembras inician su capacidad reproductiva desde los 6-9 meses de vida en el caso de las gatas (Giménez et al., 2006) y desde los 6-10 meses de vida en el caso de las perras (Buriticá et al., 2013).

Si la tendencia de esterilizaciones en la zona del estudio continúa con un mayor porcentaje de animales entre 1 y 3 años, es importante aumentar el número de campañas de esterilización al año, para así obtener un impacto poblacional a largo plazo. En Brasil, se estimó el impacto poblacional que tendrán las esterilizaciones mediante ecuaciones y tomando en cuenta dos factores: que las campañas de esterilización se realicen continuamente durante el año (80\% de tasa de esterilización) y evitar que ingresen animales de compañía de otras poblaciones; con lo cual se podría reducir en cinco años a un $20 \%$ la población de perros en el país (Amaku et al., 2009). En Italia, un estudio presentó dos opciones para disminuir la población: aumentar a un 55\% la tasa de esterilización de hembras de 3 años en promedio o disminuir la edad en la que se realice la cirugía (menos de 1 año) para que la tasa de esterilización solo sea de 26\% (Di Nardo et al., 2007).

En cuanto a la raza, hubo mayor porcentaje de animales mestizos esterilizados. Tanto perros como gatos mestizos suelen ser fáciles de adquirir mediante adopción o como regalo (Pavez, 2009). En Comas se estima la presencia de un $55.3 \%$ de perros mestizos (Soriano et al., 2014) y en San Martín de Porras de 54.1\% (Arauco et al., 2013). Es probable en Los Olivos exista un porcentaje similar de perros mestizos.

Si bien el estudio logró evaluar datos de 295 esterilizaciones de animales de compañía, no se puede determinar la proporción que representa respecto a la población total del distrito. Por ello se hace necesario realizar un estudio para la estimación de la población de perros y gatos para valorar el impacto de las estrategias de control reproductivo en estas poblaciones animales.

En líneas generales, se puede observar que los animales de compañía atendidos provienen de casi toda el área del distrito, pues el área de influencia de la Clínica Veterinaria Municipal fue de $8.3 \mathrm{~km}$, aunque hubo un agrupamiento de 65 registros (22\%) a un radio de $0.94 \mathrm{~km}$ de la clínica. Se debe considerar que los principales problemas de la inadecuada tenencia de animales de compañía se presentan en estratos poblacionales de menores niveles socioeconómicos, donde las personas no suelen tener control sobre sus animales y dejan que tanto gatos como perros deambulen por las calles con el riesgo de reproducirse en forma no controlada (Arismendy, 2010). El distrito de Los Olivos tiene una población de 371229 habitantes y el 9.7\% se encuentra en condición de pobreza (INEI, 2013); por lo tanto, las acciones asistenciales brindadas por la municipalidad deberían dirigirse principalmente a poblaciones de bajos recursos económicos, las cuales deben de ser acompañadas de sensibilización acerca de la tenencia responsable de animales de compañía.

En cuanto a los domicilios registrados, dos de ellos presentaban registros de más de ocho animales de compañía esterilizados. Al visitar los domicilios se comprobó que eran animales recogidos de la calle y que probablemente no llevaban un control sanitario adecuado, lo que puede representar un potencial riesgo para la salud pública por contaminación ambiental, transmisión de enfermedades zoonóticas y accidentes por mordedura (León et al., 2013).

El estudio muestra el incumplimiento de la normativa municipal en relación con la tenencia de animales de compañía. La Ordenanza Municipal No. 435-CDLO, en el Artículo 5 del Capítulo 1, indica que «el número 
de perros y gatos no excederá de los tres por cada domicilio; así mismo, la cantidad de más animales deberá estar acorde con las condiciones de alojamiento, que no constituyan riesgo para la salud, la tranquilidad, seguridad y bienestar de los vecinos y los animales de compañía». Esta ordenanza está basada en la Ley que regula el «Régimen Jurídico de Canes» (Ley N. ${ }^{\circ} 27596$ ), cuya finalidad es cuidar la integridad, salud y tranquilidad de las personas.

\section{Conclusiones}

- Se evaluó un total de 295 animales de compañía esterilizados, 140 perros y 155 gatos.

- El mayor porcentaje de esterilizaciones correspondieron a hembras en ambas especies.

- La edad de los perros esterilizados fue de $2.1 \pm 1.5$ y en gatos de $1.2 \pm 0.8$ años.

- El área de influencia de la Clínica Veterinaria Municipal de Los Olivos tuvo un radio de hasta $8.3 \mathrm{~km}$.

\section{Agradecimientos}

Los autores agradecen al Alcalde de la Municipalidad de Los Olivos, Pedro del Rosario Ramírez, a la Regidora María del Pilar Pérez Carbajal y al Médico Veterinario de la Clínica Veterinaria Municipal Elizabeth Cóndor Pazce.

\section{Literatura Citada}

1. Amaku M, Dias RA, Ferreira F. 2009. Dinamica populacional canina: potenciais efeitos de campanhas de esterilizac'abo. Rev Panam Salud Publ 25: 300-304.

2. Arauco D, Urbina B, León D, Falcon N. 2014. Indicadores demográficos y estimación de la población de canes con dueño en el distrito de San Martín de Porres, Lima-Perú. Salud Tecnol Vet 2: 83-92. doi: 10.20453/stv.v2i2.2254
3. Arellano $R$, Osorio $M$, Napurí MC, León D, Falcón N. 2018. Indicadores demográficos de perros y gatos con dueño en el distrito de San Borja, Lima-Perú, 2017. Salud Tecnol Vet 1: 1-8.

4. Arismendy A, Ariza O, Bautista J, Bello J, Chacon J, Delgado, Díaz M, et al. 2010. Primer censo canino y felino del área urbana del municipio de Bucaramanga, 2010. Tesis de Médico Veterinario Zootecnista. Colombia: Univ. Cooperativa de Colombia. $130 \mathrm{p}$.

5. Buriticá EF, Echeverry DF, Barbosa IX, Quintero A. 2013. Evaluación reproductiva de la hembra canina en el momento del servicio: consideraciones para la práctica clínica. Rev Colomb Cienc Anim 6: 113-117.

6. Consejo Canadiense de Protección de los Animales. 1998. Eutanasia. En: Manual sobre el cuidado y uso de los animales de experimentación. Canadá: Consejo Canadiense de Protección de los Animales. 299 p.

7. Chávez C, Falcón N, León D, Sánchez D. 2014. Canes vagabundos en el interior y alrededores de mercados formales de Villa El Salvador, Lima, Perú. Rev Inv Vet Perú 27: 176-182. doi: 10.15381/ rivep.v27i1.11456

8. Di Nardo A, Candeloro L, Budke C, Slater M. 2007. Modeling the effect of sterilization rate on owned dog population size in central Italy. Prev Vet Med 82: 308-313. doi: 10.1016/j.prevetmed. 2007.06.007

9. [FAO] Food and Agriculture Organization of the United Nations. 2014. Dog population management. Report of the FAO/WSPA/IZSAM expert meeting. Banna, Italy. Rome: FAO. Animal Production and Health Report. No. 6.61 p. [Internet]. Available in: http://www.fao.org/3/a-i4081e.pdf

10. Giménez F, Stornelli M, Savignone C, Tittarelli C, De la Sota R, Stornelli M. 2006. Fisiología reproductiva y control de los ciclos estrales en la gata doméstica. Analecta Vet 26: 38-43. 
11. Howe LM. 2006. Surgical methods of contraception and sterilization. Theriogenology 66: 500-509. doi: 10.1016/j.theriogenology.2006.04.005

12. [ICAM] International Companion Animals Management Coalition. 2007. Guía para el manejo de poblaciones caninas. ICAM. $22 \mathrm{p}$.

13. [INEI] Instituto Nacional de Estadística e Informática. 2013. Mapa de la pobreza provincial y distrital. Lima: INEI. $168 \mathrm{p}$.

14. León D, Panta S, Yarlequé C, Falcón $N$. 2013. La convivencia con mascotas en zonas periurbanas: Experiencia en Lima-Perú. MV Rev Cien Vet 29: 21-25.

15. Ley $N^{\circ}$ 27596. Ley que regula el régimen jurídico de canes. Diario Oficial "El Peruano». Lima, 14 de diciembre de 2001. [Internet]. Disponible en: https://busquedas.elperuano.pe/ normaslegales/ordenanza-que-regula-elregimen-de-tenencia-y-registro-de-caordenanza-no-385-mdsmp-1310176-1/

16. Lui J, Toniollo G, SaviI P, Voorwald F, Silva M, Tosta P. 2011. Esterilizacao cirúrgica de caninos e felinos em Jaboticabal. Interacao entre o beneficio social e a pesquisa cientifica. Rev Cienc Ext 7: 29-40.

17. Llalla H. 2012. Caracterización de la tenencia de mascotas y evaluación de conocimientos, actitudes y prácticas asociadas a riesgos de zoonosis y accidentes entre escolares de educación secundaria en tres distritos de Lima Metropolitana. Tesis de Maestría. Lima: Univ. Peruana Cayetano Heredia. 83 p.

18. Málaga H. 2010. Epidemiologia veterinaria. Lima: Univ. Peruana Cayetano Heredia. 183 p.

19. Miller D. 2012. La conexión entre la obesidad y la esterilización en los animales de compañía. Banfield J 1-6 [Internet]. Disponible en: http:// www.banfield.com.mx/pdfs/ La_Conexion_final_1P.pdf

20. Mirò̀ G, Turner D. 2003. Prevención de la superpoblacioìn canina y felina. Colegio Oficial de Veterinarios de Madrid.
[Internet]. Disponible en: http:// www.colvema.org/PDF/SUPERPOBLACION.pdf

21. Monstserrat M. 2015. Control del ciclo reproductivo en la perra y en la gata. Argos Portal Veterinario [Internet]. Disponible en: http://argos. portal veterinaria.com/noticia/12126/articulos-archivo/control-del-ciclo-reproductivo-en-laperra-y-en-la-gata.html

22. Ochoa Y, Falcon N, Suazo J, Guevara B. 2014. Estimación de la población de perros callejeros en el distrito de Los Olivos, Lima, Perú. Rev Inv Vet Perú 25: 366-373.

23. Ordenanza Municipal $N^{\circ} 435-C D L O$. 2016. Reglamento de cumplimiento y aplicación de la Ley N³0407-Ley de protección y bienestar animal, en el distrito de Los Olivos. Municipalidad distrital de Los Olivos. Los Olivos, 07 de abril de 2016. [Internet]. Disponible en: http:// portal.munilosolivos.gob.pe/ transparencia_mdlo/doc_transparencia/ Normas_Emitidas/Ordenanzas/2016/ ord-435-2016-ordenanza-que-apruebael-reglamento-de-cumplimiento-yaplicacion-de-la-ley-n-30407-ley-deproteccion-y-bienestar-animal-en-el-distrito-de-los-olivos.pdf

24. Pavez C. 2009. Estimación y algunas características de la población canina con dueño en 34 comunas de la ciudad de Santiago. Tesis de Médico Veterinario. Santiago de Chile: Univ. Iberoamericana de Ciencias y Tecnología. $30 \mathrm{p}$.

25. Reichler IM. 2009. Gonadectomy in cats and dogs: a review of risks and benefits. Reprod Domest Anim 44: 2935. doi: 10.1111/j.1439-0531.2009.01437.x

26. Rendón D, Quintana E, Door Ivanna, Vicuña F, León D, Falcón N. 2018. Parámetros demográficos en la población de canes y gatos domésticos en asentamientos humanos del distrito de Ventanilla, Callao-Perú. Rev Inv Vet Perú 29: 217-225. doi: 10.15381/rivep. v29i1. 14191 
27. Root K. 2010. Determining the optimal age for gonadectomy in dogs and cats. J Am Vet Med Assoc 231: 1665-1675.

28. Root Kustritz R. 2014. Pros, cons, and techniques of pediatric neutering. Vet Clin N Am-Small 44: 221-233. doi: 10.1016/ j.cvsm.2013.10.002

29. Root-Kustritz RV. 2012. Effects of surgical sterilization on canine and feline health and on society. Reprod Domest Anim 47: 214-222.

30. Salamanca CA, Polo LJ, Vargas J. 2011. Sobrepoblación canina y felina: tendencias y nuevas perspectivas. Rev Med Vet Zootec 58: 45-53.

31. Sontas B, Kaysigiz, F, Ekici H. 2012. Methods of oestrus prevention in dogs and cats: a survey of Turkish veterinarian's practices and beliefs. Arch Med Vet 44: 155-166. doi: 10.4067/S0301732X2012000200009
32. Soriano JF, Nuñez J, León D, Falcón N. 2017. Estimación de la población de canes con dueño en el distrito de Comas, Lima-Perú. Rev Inv Vet Perú 33: 5-10. doi: 10.15381/rivep.v25i3.10114

33. Spain CV, Scarlett JM, Houpt KA. 2004a. Long-term risks and benefits of early-age gonadectomy in cats. J Am Vet Med Assoc 224: 372-379. doi: 10.2460/ javma.2004.224.372

34. Spain CV, Scarlett JM, Houpt KA. 2004b. Long-term risks and benefits of early-age gonadectomy in dogs. J Am Vet Med Assoc 224: 380-387.

35. Tasker L. 2013. Métodos de eutanasia para perros y gatos: comparación y recomendaciones. London: WSPA. $27 \mathrm{p}$.

36. [WHO y WSPA] World Health Organization \& World Society for the Protection of Animals. 1990. Guidelines for dog population management. Geneve: WHO \& WSPA. 116 p. 